Conclusion This study confirms that differences in miRNA expression profiles between CD strictured and non-strictured areas can be detected. Upregulation of collagen mRNA shows that miR-34a might play a functional role in modulating fibrosis in $\mathrm{CD}$, however further studies to investigate the impact of increased collagen protein are required. Manipulation of miRNA profiles may be a novel therapeutic strategy against fibrosis in Crohn's disease.

Competing interests None declared.

\section{PMO-230 CLINICAL RISK FACTORS FOR CROHN'S DISEASE POSTOPERATIVE RECURRENCE ARE REFLECTED IN ALTERATIONS IN MUCOSALLY ADHERENT MICROBIOTA AT SURGICAL RESECTION}

doi:10.1136/gutjnl-2012-302514b.230

\begin{abstract}
${ }^{1,2} \mathrm{~A} \cup$ Murugananthan, ${ }^{*} \mathrm{D} O \mathrm{Bernardo},{ }^{2} \mathrm{P}$ Tozer, ${ }^{1} \mathrm{~A}$ L Hart, ${ }^{2} \mathrm{~S} \mathrm{C}$ Knight, ${ }^{3} \mathrm{~K}$ Whelan, ${ }^{1} \mathrm{~N}$ Arebi, ${ }^{2} \mathrm{H} O$ Al-Hassi. ${ }^{1}$ Gastroenterology, St Mark's Hospital, London, UK; ${ }^{2}$ Antigen Presentation Research Group, Imperial College, London, UK; ${ }^{3}$ Diabetes and Nutritional Sciences Division, KIng's College London, London, UK
\end{abstract}

Introduction Clinical risk factors for Crohn's disease (CD) recurrence after ileo-caecal resection (ICR) include smoking status, perforating disease and $>1$ surgical resection. The underlying mechanisms contributing to clinical risk are unknown. We aimed to study the relationship between risk factors and gut microbiota.

Methods Samples of macroscopically inflamed and non-inflamed small bowel from patients undergoing surgical resection for CD were analysed. Samples were snap frozen in liquid nitrogen. Cryosections were cut and the frozen sections were hybridised with oligonucleotide probes targeting the microbial 16S rRNA of total bacteria, Escherichia coli, Bacteroides-Prevotella, Faecalibacterium prausnitzii, Clostrium coccoides- Eubacterium rectale and bifidobacteria. The hybridised mucosa associated microbiota (MAM) were identified and quantified. Patients with $\geq 1$ risk factor were classified as high risk for disease recurrence.

Results Fifteen patients underwent ICR (10 female); 9 were high risk (6 smokers, 4 fistulating disease and 2 recurrent resection- 3 patients had multiple risk factors). Faecalibacterium prausnitzii numbers in inflamed operative samples were lower in smokers compared with non-smokers $(p=0.036)$. High-risk patients had lower numbers of bifidobacteria in both inflamed $(p=0.006)$ and non-inflamed $(p=0.01)$ operative samples compared with low risk patients.

Conclusion The risk of post-operative $\mathrm{CD}$ recurrence may be predetermined at a pre-operative stage due to dysbiosis. The role of MAM as a tool to stratify risk requires further study. Drugs that modulate MAM may, in future, play a role in reducing post-operative recurrence.

Competing interests None declared.

\section{PM0-231 ILEAL AND COLONIC MUCOSAL DENDRITIC CELL CYTOKINE PROFILES DIFFER AT REST AND AFTER IN VITRO BACTERIA AND PRO-BIOTIC CHALLENGE IN POSTOPERATIVE CROHN'S DISEASE PATIENTS}

doi:10.1136/gutjnl-2012-302514b.231

\footnotetext{
${ }^{1,2} \mathrm{~A}$ U Murugananthan, ${ }^{*}{ }^{2} \mathrm{D} O$ Bernardo, ${ }^{2} \mathrm{E}$ R Mann, ${ }^{1} \mathrm{C}$ T Tee, ${ }^{1} \mathrm{~A}$ L Hart, ${ }^{1} \mathrm{~N}$ Arebi, ${ }^{2} \mathrm{~S}$ C Knight, ${ }^{2} \mathrm{H} O$ Al-Hassi. ${ }^{1}$ Gastroenterology, St Mark's Hospital, London, UK; ${ }^{2}$ Antigen Presentation Research Group, Imperial College, London, UK
}

Introduction Postoperative Crohn's disease (CD) recurrence predominantly affects the ileal mucosa at the ileo-colonic anastomosis with the colonic side often spared. Altered immune responses to bacterial flora are thought to be a driving force in the patho- genesis of CD recurrence. Gut dendritic cells (DC) are key in the initiation of immune response, through cytokine production, when stimulated with bacterial antigens. We postulate that differences between ileal and colonic DC resting characteristics and functional responses may be responsible for the propensity of recurrence to occur at the ileal aspect of the anastomosis. We aimed to assess ongoing intracellular cytokine production in DC from ileal and colonic postoperative $\mathrm{CD}$ mucosa and assess their functional response to bacterial stimulation and modulation with probiotics.

Methods Paired ileal and colonic biopsies were taken from postoperative CD patients at colonoscopy $(n=11)$. Lamina propria mononuclear cells were collected after collagenase digestion. DC intracellular cytokine responses (IL-2, IL-6, IL-17a, TGF $\beta$ and INF $\gamma$ ) were assessed in basal conditions and after culture with LPS and two probiotic bacterial strains Bifidobacterium Longum; Lactobacillus Casei (B longum and $L$ case $i$ ) using multi-colour flow cytometry.

Results Unstimulated ileal DC showed higher levels of ongoing intracellular production of pro-inflammatory cytokines than unstimulated colonic DC: IL6 $(34.21 \pm 12.80$ vs $10.47 \pm 3.574$ cells $/ u l$ [mean \pm SEM], $\mathrm{p}=0.037)$, IL17a $(24.62 \pm 12.38$ vs $14.94 \pm 9.865$ cells/ Ul, $\mathrm{p}=0.05, \mathrm{n}=5)$ and TGF $\beta(74.12 \pm 17.96$ vs $32 \pm 16.27$ cells $/ \mathrm{ul}$, $\mathrm{p}=0.031$ ). Incubation with LPS resulted in higher DC intracellular cytokine levels of INF $\gamma$ in ileal derived DC with a borderline $p$ value $(27.49 \pm 12.61$ vs $0.39 \pm 0.391$ cells $/ v 1 \mathrm{p}=0.06)$ but not colonic derived DCs $(19.55 \pm 10.12$ vs $12.40 \pm 7.039, \mathrm{p}=0.6)$. L casei incubation, however, led to a larger decrease in ongoing TGF $\beta(-42.35 \pm 16.02$ vs $4.42 \pm 11.46$ cells $/ \mathrm{ul} p=0.023)$ and INF $\gamma \quad(-14.76 \pm 7.196$ vs $20.33 \pm 10.16$ cells $/$ ul-, $p=0.05)$ DC cytokine production in colonic tissue compared with ileal.

Conclusion Ileal mucosa DC demonstrate a cytokine profile implicating a Th17 response compared with colonic mucosa. Upon bacterial stimulation with LPS ileal mucosa demonstrate increased INF $\gamma$ DC production compared with unstimulated DC. These results suggest a role of for a Th1/Th17 response in driving postoperative $\mathrm{CD}$ recurrence. The probiotics $L$ casei and $B$ longum failed to show significant effects in modulation of intracellular cytokine production in ileal DC.

Competing interests None declared.

\section{PM0-232 ABNORMAL LIVER FUNCTION TEST IN PATIENTS WITH ULCERATIVE COLITIS: A RETROSPECTIVE STUDY}

doi:10.1136/gutjnl-2012-302514b.232

A A Thi, ${ }^{*}$ K Holbrook, R Makins. Department of Gastroenterology, Cheltenham General Hospital, Cheltenham, UK

Introduction The association between primary sclerosing cholangitis (PSC) and ulcerative colitis (UC) is well recognised. The prevalence of PSC in patients with UC has been reported widely and ranges from $2.4 \%$ to $7.5 \%$. The mean annual incidence rates were between 0.9 and 1.3 cases per 100000 person years. Patients with UC may frequently be found to have abnormal liver biochemistry (LFTs) for numerous reasons although PSC is uncommon. Given the known increased risk of colorectal cancer in patients with both UC and PSC as well as cholangiocarcinoma, early recognition of PSC is crucial. Methods Aims: To identify known patients with UC from our clinic population who also had persistently elevated LFTs and to determine the extent to which the cause of the abnormal LFTs had been investigated.

Methods A representative sample of patients with UC was identified from those who had contacted the nurse led IBD telephone help line at Gloucestershire Hospitals NHS Foundation Trust during September and October 2010. UC diagnosis was based on histology proven on biopsies including colectomy. Abnormal LFTs were defined as a persistent elevation above the local laboratory upper 
limit of normal (ALT > $40 \mathrm{IU} / 1$, ALP $>200 \mathrm{IU} / 1$, GGT $>30 \mathrm{IU} / 1$ and bilirubin $>17 \mathrm{mmol} / \mathrm{l})$. The investigations performed to identify the cause of the abnormalities were noted.

Results A total of 450 phone calls were made to the IBD help line during the study period. Of those, 82 patients with ulcerative colitis were identified. Of these, 15 patients (18.3\%) had so far been found to have abnormal LFTs at some time during the course of their illness. Persistently abnormal LFTs were identified in 10 patients $(12.2 \%)$. Of these 10, $6(60 \%)$ had auto immune screen, four patients (40\%) had viral hepatitis screen, four patients (40\%) had a liver ultrasound, three patients $(30 \%)$ had CT abdomen. Furthermore no patients had targeted investigations to exclude PSC such as a liver biopsy or magnetic resonance cholangiography.

Conclusion Our results suggest that $12 \%$ of ulcerative colitis patients in our cohort had persistently abnormal liver function tests although none had had further investigations for PSC. Given expected prevalence data we could perhaps expect to see one patient with PSC in this cohort. The monitoring and following of LFTs in patients with UC should be part of standard follow-up procedures.

Competing interests None declared.

\section{REFERENCES}

1. Chapman R, Fevery J, Kalloo A, et al. Diagnosis and management of primary sclerosing cholangitis. Hepatology 2010;51:600-78.

2. Tishendorf JJW, Geier A, Trautwein C. Current diagnosis and management of primary sclerosing cholangitis. Liver Transplantation 2008;14:735-46.

\section{PMO-233 A PROSPECTIVE AUDIT OF THE USE OF CALCINEURIN INHIBITORS IN PATIENTS WITH REFRACTORY ULCERATIVE PROCTO-COLITIS IN A DISTRICT GENERAL HOSPITAL}

doi:10.1136/gutjnl-2012-302514b.233

B D Warner, ${ }^{*}$ L Blackmore, C Mungalsingh, A W Harris. Gastroenterology, Maidstone and Tunbridge Wells NHS Trust, Tunbridge Wells, UK

Introduction Calcineurin inhibitors (CI), most commonly ciclosporin, may be used as salvage therapy in patients with refractory moderate-severe ulcerative procto-colitis (UC), who would otherwise require surgery. ${ }^{1}$ However, use of CI may be limited by drug toxicity. For patients who have a good clinical response to ciclosporin but experience side effects, tacrolimus may be used as an alternative. ${ }^{2}$ We report our experience with $\mathrm{CI}$ in patients with moderate-severe UC whom either failed, or were intolerant of, thiopurines and as an alternative to surgery.

Methods NICE (January 2008) did not support the use of infliximab in patients with moderate-severe UC. ${ }^{3}$ Our patients were offered the choice of surgery or treatment with CI. Clinical response was assessed by $\mathrm{AWH}$ in clinic. If ciclosporin $(4-6 \mathrm{mg} / \mathrm{kg} / \mathrm{day}$ in two divided doses) led to a clinical response but caused intolerable side effects, tacrolimus $(0.1 \mathrm{mg} / \mathrm{kg} /$ day in two divided doses) was offered as an alternative. Failure of therapy prompted referral for surgery.

Results 14 patients ( 8 female; mean age 38 [range 22-56] years) were treated with CI (13 ciclosporin, 1 tacrolimus). Ten of 14 (71\%) patients had an initial clinical response to CI. Adverse effects were common (57\%): nausea, paraesthesia, menstrual disturbance, maculopapular rash, hypertension (two patients) and renal dysfunction (one patient). Of the four patients who failed to respond to $\mathrm{CI}$, three were referred for surgery and one patient is managed on mesalazine suppositories. A further four patients stopped treatment with CI due to adverse effects: two were referred for surgery and two were offered treatment with methotrexate. 6 (43\%) of 14 patients responded successfully to CI and without side effects. $4(30 \%)$ of these remain on CI: 3 on ciclosporin (mean duration of treatment 39 [range 19-71] months) and one patient on tacrolimus (duration of treatment 24 months); two patients stopped treatment with $\mathrm{CI}$ for reasons unrelated to efficacy or adverse effects (one to start a family and the other out of choice).

Conclusion CI should be considered as an alternative therapy for patients with refractory moderate-severely active UC who would otherwise require surgery. CI can be used safely and effectively in the presence of an established evidence-based protocol to ensure safe prescribing and monitoring for adverse side effects.

Competing interests None declared.

\section{REFERENCES}

1. Lichtiger S, Present $\mathrm{DH}$, Kornbluth $\mathrm{A}$, et al. Cyclosporine in severe ulcerative colitis refractory to steroid therapy. N Eng J Med 1994;330:1841-5.

2. Ogata H, Matsui $T$, Nakamura $M$, et al. A randomised dose finding study of oral tacrolimus (FK506) therapy in refractory ulcerative colitis. Gut 2006;55:1255-62.

3. Ulcerative colitis (subacute manifestations)-Infliximab (TA140) NICE.

\section{PM0-234 IS THE RUTGEERTS' SCORE OF ANY CLINICAL VALUE AFTER ILEO-COLIC RESECTION FOR CROHN'S DISEASE? A PROSPECTIVE STUDY IN DISTRICT GENERAL HOSPITAL PRACTICE}

doi:10.1136/gutjnl-2012-302514b.234

B D Warner, ${ }^{*}$ L Blackmore, P Blaker, A Harris. Gastroenterology, Maidstone and Tunbridge Wells NHS Trust, Tunbridge Wells, UK

Introduction Disease recurrence after surgical resection for Crohn's disease (CD) is observed in $20 \%-30 \%$ of patients at 1 year, with a $10 \%$ increase per year in subsequent years. The European Crohn's and Colitis Organisation currently recommends ileo-colonoscopy 1 year after ileo-colic resection, since this predicts the need for further surgery (Statement 8C) within 2 years. ${ }^{1}$ This statement is not supported by the BSG guidelines, highlighting a need for prospective studies to determine the role of the Rutgeert's score following ileo-colic resection. The aim of this study was to determine if the Rutgeert's score ${ }^{3}$ at $1-2$ years after ileo-colic resection predicts clinical recurrence and/or need for further surgery in patients with $\mathrm{CD}$ in district general hospital practice.

Methods Between 2005 and 2011, 43 patients with fibrostenotic or penetrating terminal ileal or right sided CD underwent ileo-colic resection. Ileo-colonoscopy was performed in 34 asymptomatic patients between 1 and 2 years following surgery. A single expert observer (AWH) assessed the surgical anastomosis to determine the Rutgeerts' score $^{3}\left(\mathrm{i}_{0}-\mathrm{i}_{4}\right)$. Nine patients who underwent resection were excluded either because ileo-colonoscopy was unsuccessful or the patient refused endoscopic assessment.

Results 14 of 15 (93\%) patients with Rutgeert's scores $i_{0}$ or $i_{1}$ remained asymptomatic from CD (Harvey Bradshaw Index $\leq 4$ ) at January 2012 (range of follow-up 4-69 months, mean of 30 months after ileo-colonoscopy). Three (20\%) of these patients smoked. Of 19 patients scoring $i_{2}$ to $i_{4}, 12$ (63\%) had clinical recurrence requiring medical treatment with immunosuppression and/or biologics. $47 \%$ of patients with a Rutgeert's score of $12-\mathrm{i} 4$ and $50 \%$ of those with clinical recurrence were current smokers.

Conclusion In district general hospital practice, a low Rutgeert's score $\left(i_{0}\right.$ or $\left.i_{1}\right)$ at $1-2$ years after ileo-colic resection for CD predicts prolonged clinical remission without the need for medical treatment. By contrast, in those patients with a Rutgeert's scores $\geq i_{2}$ clinical recurrence occurred within a maximum of 16 months following surgical resection with a higher rate of recurrence among smokers. 